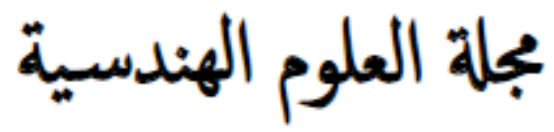

\title{
Numerical study on a new design of solid-fuel ramjet combustor with swirl flow
}

\author{
Omer Musa ${ }^{1,3,},{ }^{3}$ Xiong Chen ${ }^{2}$ and Guoping Huang ${ }^{1}$ \\ 1 College of Energy and Power Engineering, Nanjing University of Aeronautics and Astronautics, Nanjing 210016, P. R. China \\ School of Mechanical Engineering, Nanjing University of Science and Technology, Nanjing 210094, P. R. China \\ Mechanical Engineering Department, Omdurman Islamic University, Omdurman, Sudan \\ * Corresponding author: Omer Musa (e-mail: omermusa@nuaa.edu.cn ).
}

Article history: Received xx January 2019, Received in revised form 05 July 2019, Accepted 05 October 2019

\begin{abstract}
A new design of solid-fuel ramjet is proposed and examined numerically in this paper. Multi-physics coupling code is developed using FORTRAN and parallel computing to solve the problems of multi-physics coupling of fluid mechanics, solid pyrolysis, heat transfer, thermodynamics, and chemical kinetics. Simulations are carried out for the proposed design then the results are compared with the classic design of the solid-fuel ramjet. It is found that the proposed design has improved the regression rate significantly; besides, the amount of released solid fuel is increased for the same size. A new flame has been observed inside the combustion chamber of the proposed design then the two flames were merged in the afterburning chamber.
\end{abstract}

Keywords: Solid fuel, Ramjet, CFD, Combustion characteristics.

\section{INTRODUCTION}

The birth of ramjet is attributed to Lake, who issued a patent for ramjet propulsion in 1909 $[1,2]$. Renee Leduc successfully flight tested a ramjet propulsion system through the Leduc 010, while requiring support from a transport aircraft to attain sufficient flight velocity and altitude [3]. Given the inherent design, the ramjet is not capable of producing static thrust, since the standard ramjet requires compressible airflow through the inlet [1]. Traditional rocket motor requires carrying an oxidizer which makes the rocket heavier and then decreases its flight range. For the same size of rocket motor, solid-fuel ramjet (SFRJ) could flight for longer range with much higher speed since it uses its forward motion to draw in oxygen from surrounding atmosphere. Therefore, the larger fuel store allows SFRJ to sustain high speed during flight.
Besides, SFRJ is a simple air-breathing engine, in design, that contains no moving parts. Thus, the internal design of the SFRJ determines its performance. However, low fuel regression rate is the main disadvantage of the SFRJ, usually less than one $\mathrm{mm} / \mathrm{sec}[4]$, which is the key parameter in the design and operation of SFRJs that is primarily depend on the heat transfer from the flame to the fuel surface.

In open literature, few studies are available regarding different solid-fuel ramjet combustor's designs, though, complex configurations have been investigated extensively in hybrid rocket motors. Lee et al. [5] studied the regression rate characteristics of helical grain. A higher regression rate is achieved when the pitch number is small, and the combination of helical grain with a large pitch number and a moderate inlet swirl may produce the maximum regression rate. Li et al. [6] investigated numerically the 


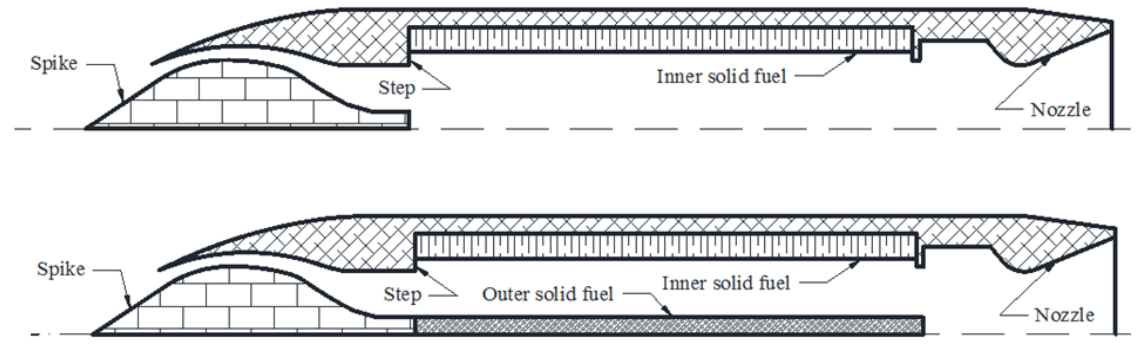

Fig. 1. The classic (upper) and proposed (lower) design of solid-fuel ramjet.

regression rate distributions for tube, star, and wagon wheel grains. The regression rates of star and wagon wheel grains were generally higher than that of tube grain under the same oxidizer mass flux.

The classical design of SFRJ basically consists of air intake system, combustion chamber, and nozzle in which the combustor contains flame-holder and cylindrical solid fuel grain (see Fig. 1 - upper). However, in order to overcome the disadvantage of SFRJ and enhance its combustion characteristics and performance we proposed and studied a new SFRJ design which contains additional solid fuel (rod) at the center of the tubular grain (see Fig. 1 - lower). The idea is to increase the solid fuel surface area contacting with incoming air to enhance the heat transfer to the solid fuel and then the regression rate. Besides, maintaining the amount of solid fuel released during flight which makes the thrust almost stable.

In this paper, the proposed SFRJ design is investigated numerically using an in-house CFD code. The code is written by FORTRAN with parallel computing to solve the problems of multi-physics coupling of fluid mechanics, solid pyrolysis, heat transfer, thermodynamics, and chemical kinetics.

\section{MATHEMATICAL MODELING}

\section{A. Governing equations}

The flow field considered is essentially three dimensional (3-D) for which all velocity and Reynolds stress components are included with regard to the simplifying condition of symmetry, whereas the flow is still angular symmetry $(\partial / \partial \theta=0)$. Therefore, the 3-D Reynolds-average Navier-Stokes equations with angular symmetry condition in integral form, which are representing the conservation of mass, momentum, energy, and species equations, can be written as:

$$
\begin{aligned}
& \frac{\partial}{\partial t} \iiint_{\Omega} \boldsymbol{U} d \Omega+\iint_{s}\left(\boldsymbol{F} \cdot n_{x}+\boldsymbol{G} \cdot n_{y}\right) d s- \\
& \iint_{s}\left(\boldsymbol{F}_{v} \cdot n_{x}+\boldsymbol{G}_{v} \cdot n_{y}\right) d s=\iiint_{\Omega}\left(\boldsymbol{H}+\boldsymbol{H}_{v}\right) \cdot \varepsilon d \Omega+\boldsymbol{S}
\end{aligned}
$$

where $\boldsymbol{n}=n_{x} \boldsymbol{i}+n_{y} \boldsymbol{j}$ is the unit normal vector in outward direction of the boundary surface $s, t$ is the time, $\varepsilon=0$ for two-dimensional plane flow, and $\varepsilon=1$ for axisymmetric flow. The conservative vector $\boldsymbol{U}$, convective flux vectors $\boldsymbol{F}, \boldsymbol{G}$, viscous flux vectors $\boldsymbol{F}_{v}, \boldsymbol{G}_{v}$, axisymmetric source terms $\boldsymbol{H}, \boldsymbol{H}_{v}$, and chemical reactions source term $\boldsymbol{S}$ are given by:

$$
\boldsymbol{U}=\left[\begin{array}{c}
\rho \\
\rho u \\
\rho v \\
\rho w \\
\rho E \\
\rho_{i}
\end{array}\right], \boldsymbol{F}=\left[\begin{array}{c}
\rho u \\
\rho u^{2}+p \\
\rho u v \\
\rho u w \\
(\rho E+p) u \\
\rho_{i} u
\end{array}\right], \boldsymbol{G}=\left[\begin{array}{c}
\rho v \\
\rho v u \\
\rho v^{2}+p \\
\rho v w \\
(\rho E+p) v \\
\rho_{i} v
\end{array}\right]
$$

$\boldsymbol{G}_{v}=\left[\begin{array}{c}0 \\ \tau_{y x} \\ \tau_{y y} \\ \tau_{y \theta} \\ u \tau_{y x}+v \tau_{y y}+w \tau_{y \theta}+q_{y} \\ \rho D_{i} \frac{\partial c_{i}}{\partial y}\end{array}\right], \boldsymbol{H}=\frac{-1}{y}\left[\begin{array}{c}\rho v \\ \rho v u \\ \rho\left(v^{2}-w^{2}\right) \\ 2 \rho v w \\ (\rho E+p) v \\ \rho_{i} v\end{array}\right]$

$$
\boldsymbol{H}_{v}=\frac{1}{y}\left[\begin{array}{c}
0 \\
\tau_{y x} \\
\tau_{y y}-\tau_{\theta \theta} \\
2 \tau_{y \theta} \\
u \tau_{y x}+v \tau_{y y}+w \tau_{y \theta}+q_{y} \\
\rho D_{i} \frac{\partial c_{i}}{\partial y}
\end{array}\right], \boldsymbol{S}=\left[\begin{array}{c}
s_{\rho} \\
s_{\rho u} \\
s_{\rho v} \\
s_{\rho w} \\
s_{\rho E} \\
\dot{\omega}_{i}+s_{\rho i}
\end{array}\right]
$$

and 


$$
\begin{gathered}
\tau_{x x}=\frac{2}{3} \mu\left(2 \frac{\partial u}{\partial x}-\frac{\partial v}{\partial y}-\frac{v}{y}\right), \tau_{y y}=\frac{2}{3} \mu\left(2 \frac{\partial v}{\partial y}-\frac{\partial u}{\partial x}-\frac{v}{y}\right), \\
\tau_{x y}=\tau_{y x}=\mu\left(\frac{\partial u}{\partial y}+\frac{\partial v}{\partial x}\right), \tau_{y y}-\tau_{\theta \theta}=2 \mu\left(\frac{\partial v}{\partial y}-\frac{v}{y}\right) \\
\tau_{x \theta}=\mu\left(\frac{\partial w}{\partial x}\right), \tau_{y \theta}=\mu\left(\frac{\partial w}{\partial y}-\frac{w}{y}\right), \\
q_{x}=k \frac{\partial T}{\partial x}+\rho \sum_{i=1}^{N} D_{i} h_{i} \frac{\partial c_{i}}{\partial x}, q_{y}=k \frac{\partial T}{\partial y}+\rho \sum_{i=1}^{N} D_{i} h_{i} \frac{\partial c_{i}}{\partial x}, \\
i=1 \rightarrow N
\end{gathered}
$$

where $u, v, w, T, p, \rho$, and $E$ represent the axial, radial, tangential velocities, temperature, pressure, total density, and total energy per unit mass, respectively; $s_{\rho}, s_{\rho u}, s_{\rho v}, s_{\rho w}$, and $s_{\rho E}$ are the mass, momentum, and energy source terms due to solid fuel pyrolysis and chemical reaction at the first cell in the fluid domain attached with the solid fuel, respectively. The variable $\dot{\omega}_{i}$ is the mass production rate of species $i$ due to chemical reactions, $c_{i}=\rho_{i} / \rho$ is the mass fraction, $N$ is the number of species, $\tau$ is the shear stress, $k$ is the thermal conductivity coefficient of the gases, and $\mu$ is the total effective viscosity which equals to the summation of laminar $\mu_{1}$ and turbulent $\mu_{t}$ viscosities. The laminar viscosity of the mixture is calculated using the following formula:

$$
\mu_{1}=\sum_{i=1}^{N} \frac{X_{i} \mu_{1 i}}{\phi_{i}}, \phi_{i}=\sum_{j=1}^{N} \frac{X_{j}\left[1+\sqrt{\left.\frac{\mu_{1 i}}{\mu_{1 i}}\left(\frac{M_{i}}{M_{j}}\right)^{1 / 4}\right]^{2}}\right.}{\sqrt{8\left(1+\frac{M_{i}}{M_{j}}\right)}}
$$

where $\mu_{l i}, M_{i}$, and $X_{i}$ are the laminar viscosity, molecular weight, and mole fraction of species $i$, respectively. The mass diffusivity $D_{i}$ of species $i$ in the mixture is given by:

$$
\rho D_{i}=\frac{1-X_{i}}{1-c_{i}}\left(\frac{\mu_{1}}{S_{c}}+\frac{\mu_{\mathrm{t}}}{S_{c \mathrm{t}}}\right)
$$

with the laminar and turbulent Schmidt numbers are assumed constant $S_{c}=0.5, S_{c t}=0.8$, respectively. The total energy per unit volume is determined using Eq. (4) and then used to calculate the temperature using Newton iteration method.

$$
\begin{aligned}
& \rho E-\frac{1}{2} \rho\left(u^{2}+v^{2}+w^{2}\right) \\
& =\sum_{i=1}^{N} \rho_{i}\left(\int_{298}^{T} C_{p i} d T+h_{i}^{298}\right)-R_{u} T \sum_{i=1}^{N} \frac{\rho_{i}}{M_{i}}
\end{aligned}
$$

where $h_{i}^{298}$ is the heat of formation at reference temperature $(298 \mathrm{~K})$, and at high temperatures the specific heat at constant pressure $C_{p i}$ is estimated by:

$$
C_{p i}=\left(a_{1 i}+a_{2 i} T+a_{3 i} T^{2}+a_{4 i} T^{3}+a_{5 i} T^{4}\right) R_{u}
$$

coefficients $a_{j i}$ are obtained from the chemical kinetics package and $R_{u}$ is the universal gas constant. For thermally perfect gases the equation of state is given by:

$$
p=\sum_{i=1}^{N} \frac{\rho_{i}}{M_{i}} R_{u} T
$$

For the mixture, the total density $\rho=\sum_{i=1}^{N} \rho_{i}$, mixture gas constant $R=R_{u} \sum_{i=1}^{N} c_{i} / M_{i}$, the specific heat $C_{p}=\sum_{i=1}^{N} c_{i} C_{p i}$, and the ratio of total specific heats $\gamma=C_{p} /\left(\mathrm{C}_{p}-\mathrm{R}\right)$.

\section{B. Finite rate model}

In this work, finite rate model is adopted to represent the chemical reaction processes. For reactions, the reaction mechanism is presented by:

$$
\sum_{i=1}^{N} v_{i k}^{\prime} X_{i} \rightleftharpoons \sum_{i=1}^{N} v_{i k}^{\prime \prime} X_{i}, k=1, \ldots, I
$$

where $v_{i k}^{\prime}$ and $v_{i k}^{\prime \prime}$ are stoichiometric coefficients. The rate of mass production of species $N$ in $I$ reactions is expressed as:

$$
\dot{\omega}_{i}=M_{i} \sum_{k=1}^{I}\left(v_{i k}^{\prime \prime}-v_{i k}^{\prime}\right)\left[R_{f}-R_{b}\right]
$$

and

$$
\begin{aligned}
& R_{f}=\dot{r}_{f_{k}} \prod_{i=1}^{N}\left(\frac{\rho_{i}}{M_{i}}\right)^{v_{i k}} \cdot\left(\sum_{j=1}^{N} \frac{\rho_{j}}{M_{j}} C_{j}\right)^{L_{M}}, \\
& R_{b}=\dot{r}_{b_{k}} \prod_{i=1}^{N}\left(\frac{\rho_{i}}{M_{i}}\right)^{v_{i k}} \cdot\left(\sum_{j=1}^{N} \frac{\rho_{j}}{M_{j}} C_{j}\right)^{L_{M}}
\end{aligned}
$$

where $\dot{r}_{f_{k}}$ and $\dot{r}_{b_{k}}$ are the forward and backward reaction rate constants for each reaction, respectively; $C_{j}$ is the third-body efficiency; 
$L_{M}=1$ when there is the third-body $(M)$ and $L_{M}=0$ elsewhere. Arrhenius formula (Eq. 9) is utilized to determine reaction rate constants

$$
\dot{r}_{k}=A_{k} T_{\mathrm{w}}{ }^{n_{k}} \exp \left[-\left(E_{a}\right)_{k} / R_{u} T_{\mathrm{w}}\right]
$$

where $A_{k}$ is the pre-exponential factor, $n_{k}$ is the temperature exponent, $E_{a}$ is the activation energy, and $T_{\mathrm{w}}$ is the solid-fluid interface wall temperature. These coefficients are taken from [7] to HDPE solid fuel. In order to model the solid fuel pyrolysis in the solid-fluid interface the zeroth order $\left(n_{k}=0\right)$ Arrhenius equation is used. The mass rate of fuel freed by pyrolysis is determined using:

$$
\dot{m}=\rho_{\text {sol }} \dot{r}=\rho_{\text {sol }} A_{\text {sol }} \exp \left[-\left(E_{a}\right)_{\text {sol }} / R_{u} T_{\mathrm{w}}\right]
$$

The subscript "sol" indicates the solid fuel.

Since the High Density Polyethylene (HDPE) grain is selected to be a solid fuel for the SFRJ. Therefore, the gas-phase chemical reactions kinetic of HDPE $[8,9]$ can be written as:

$$
\left.\begin{array}{l}
\mathrm{C}_{2} \mathrm{H}_{4}+\mathrm{O}_{2} \rightarrow 2 \mathrm{CO}+2 \mathrm{H}_{2} \\
2 \mathrm{CO}+\mathrm{O}_{2} \rightarrow 2 \mathrm{CO}_{2} \\
2 \mathrm{H}_{2}+\mathrm{O}_{2} \rightarrow 2 \mathrm{H}_{2} \mathrm{O}
\end{array}\right\}
$$

where the number of reactions are three $(I=3)$ with six species $(N=6)$.

\section{Governing equations of solid domain}

The heat diffusion in the solid domain is modeled by reducing RANS equation (Eq. (1)) to Fourier's equation (Eq. (12)) by evaluating all velocities to zeros and considering only one of the species (solid). The two-dimensional/axisymmetric Fourier equation can be written in integral form as:

$$
\begin{aligned}
& \frac{\partial}{\partial t} \iiint_{\Omega} \boldsymbol{U}^{\text {sol }} d \Omega-\iint_{s}\left(\boldsymbol{F}_{v}^{\text {sol }} \cdot n_{x}+\boldsymbol{G}_{v}^{\text {sol }} \cdot n_{y}\right) d s \\
& =\varepsilon \iiint_{\Omega} \boldsymbol{H}_{v}^{\text {sol }} d \Omega+\boldsymbol{S}^{\text {sol }}
\end{aligned}
$$

and

$$
\begin{gathered}
\boldsymbol{U}^{\text {sol }}=\rho_{\text {sol }} c_{\text {sol }} T_{\text {sol }}, \boldsymbol{F}_{v}^{\text {sol }}=k_{\text {sol }} \frac{\partial T_{\text {sol }}}{\partial x}, \boldsymbol{G}_{v}^{\text {sol }}=k_{\text {sol }} \frac{\partial T_{\text {sol }}}{\partial y}, \\
\boldsymbol{H}^{\text {sol }}=\frac{1}{y} k_{\text {sol }} \frac{\partial T_{\text {sol }}}{\partial y}, \boldsymbol{S}^{\text {sol }}=\rho_{\text {sol }} \dot{r} h_{p}
\end{gathered}
$$

where $\rho_{\text {sol }}, k_{\text {sol }}$, and $c_{\text {sol }}$ denote the density, thermal conductivity, and specific heat capacity of the solid material, respectively. The specific heat capacity of HDPE fuel is taken from [10]; $T_{\text {sol }}$ is the temperature in the solid domain, $h_{p}$ is the reaction energy per unit mass, and $S_{\text {sol }}$ is the source term due to the chemical reaction of the burning surface attached with the fluid domain

\section{BOUNDARY CONDITIONS}

The boundary conditions are necessary to fully define the flowfield beside the governing equations. At the inlet boundary, the axial, and swirl velocities profiles are given by the measurement data. At the axis, axisymmetric boundary condition is employed. At the exit, all axial gradients of flow quantities are set to zero. For walls boundaries, no-slip adiabatic boundary condition is used. In addition, at the solid-fluid interface wall the energy balance is conducted to determine the wall temperature $T_{w}$. The energy balance equation at interface is derived by [10]:

$$
\left.k \frac{\partial T}{\partial y}\right|_{\text {gas }}=-\left.k_{\text {sol }} \frac{\partial T}{\partial y}\right|_{\text {solid }}
$$

\section{NUMERICAL SOLUTION}

For the fluid domain, the prescribed RANS equations have been solved simultaneously by means of cell-centered, finite-volume method with structured grids, multi-block, and density-based approach. At each cell of the SFRJ flow field meshes (104982 cells) the governing equations are discretized and numerically solved in which AUSMPW+ [11], third-order upwind-based MUSCL [12], and Van albada limiter function [13] are employed to evaluate inviscid fluxes with the second-order central differences for viscous fluxes. The turbulence is treated by Menter's shear-stress transport (SST) turbulence model [14]. Finite rate model is employed to determine the chemical source terms. In this model the flame is treated as laminar flame with neglecting the effect of turbulent fluctuating (turbulence-chemistry interaction), at which Arrhenius expression is 
used to determine the mean reaction rate. Since we considered unsteady simulations, the lower-upper symmetric Gauss-Seidel (LU-SGS) implicit dual time-stepping algorithm is selected for fluid domain and backward Euler method for solid domain [15].

In this work, two cases are simulated with different combustor designs, the proposed design and the classic one. The total temperature is set to be $540 \mathrm{~K}$ and the mass flow rate is fixed at $0.6 \mathrm{~kg} / \mathrm{s}$. Other boundary conditions are treated as follows; no-slip adiabatic boundary condition at walls, axisymmetric boundary condition at axis, all axial gradient quantities are set to zero at exit, and at solid-fluid interface the solid and fluid domains are coupled by direct coupling approach in which the wall temperature is determined by energy balance equation.

\section{THE CODE STRUCTURE AND SIMULATION PROCEDURE}

A multi-physics coupling code is developed to solve the problems of mathematical modeling mentioned in previous sections of multi-physics coupling of fluid mechanics, solid pyrolysis, heat transfer, thermodynam ics, and chemical kinetics. The developed code contains a broad physical modeling capabilities needed to model flow, turbulence, heat transfer, solid pyrolysis, and combustion (see Fig. 2). OpenMP (Open Multi-Processing) programming interface is used to share memory in parallel computers. The code is written using FORTRAN language and the simulations were done on cluster computer.

In Fig. 1, the ramjet has inlet inner diameter of $40 \mathrm{~mm}$, grain port inner diameter of $70 \mathrm{~mm}$, grain length of $300 \mathrm{~mm}$. The diaphragm diameter is $62 \mathrm{~mm}$ and aft-mixing chamber diameter is $74 \mathrm{~mm}$. The nozzle throat diameter is $36 \mathrm{~mm}$ and the exit nozzle diameter is $53 \mathrm{~mm}$, the dimensions of which are based on a typical experimental test engine. The mesh comprised of a multi-block 2-D structured grids with total mesh number 104982 cells and the first cell height from the wall is 10$6 \mathrm{~m}$ which gives $\mathrm{y}^{+} \approx 1$. The rod is $20 \mathrm{~mm}$ diameter and $303 \mathrm{~mm}$ in length. The mesh is clustered along the walls, and near the shear layer region to resolve accurately the flame stabilization point and the steep gradients. In solid domain, the first cell size from the interface wall is set to be fine enough to ensure grid independence of the heat flux estimation and to resolve accurately the temperature gradient. To examine the grid sensitivity, in addition to the adopted grids, we also carried out numerical simulations for swirling reacting flows on coarsest and finest meshes. No significant difference for all of the flow parameters were obtained between the finest and adopted meshes. The total temperature is set to be $540 \mathrm{~K}$ and the mass flow rate is fixed at $0.6 \mathrm{~kg} / \mathrm{s}$.

An extensive verification and validation study has been performed on the base code in our previous work [16], including uncertainty and numerical errors. In addition, the base code has been also validated using swirl benchmark cases in the previous work [17]. Furthermore, the extended code with combustion model needs to be validated on combustion cases, to do so; additional two cases have been studied as well in [18-20].

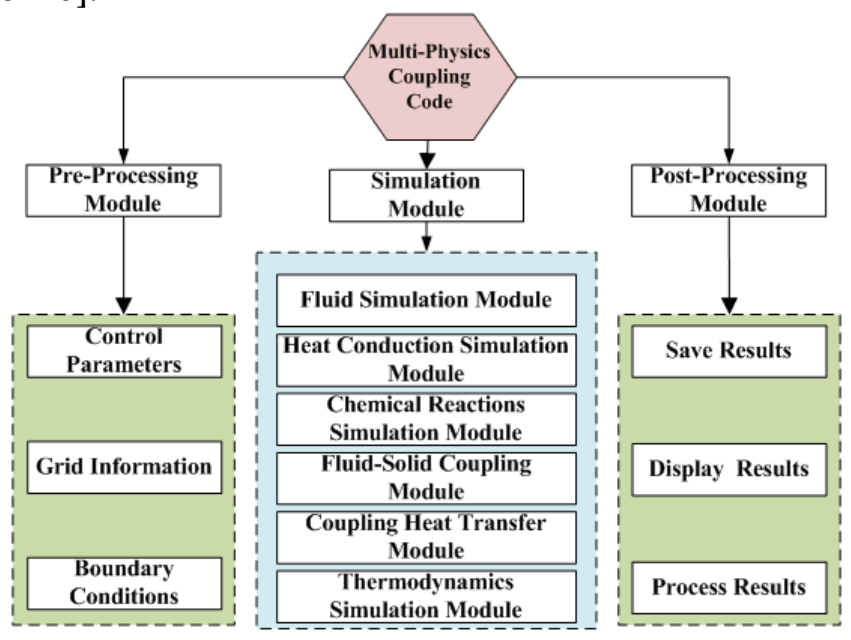

Fig. 2. Multi-physics coupling code modules.

\section{RESULTS AND DISCUSSION}

For the prescribed cases, the numerical simulation results are presented in this section. The ignition process is achieved by using hot gas 
flowing together with the incoming air into the combustor. The ignition gas flows with $0.4 \mathrm{~kg} / \mathrm{s}$, total temperature of $2500 \mathrm{~K}$ and contains $\mathrm{N}_{2}, \mathrm{HO}_{2}$, and $\mathrm{CO}_{2}$. The ignition gas is produced through combustion of double-based propellant and only the gas components are considered in the model. After 0.35 second the ignition gas has stopped and only the incoming air flow is continued.

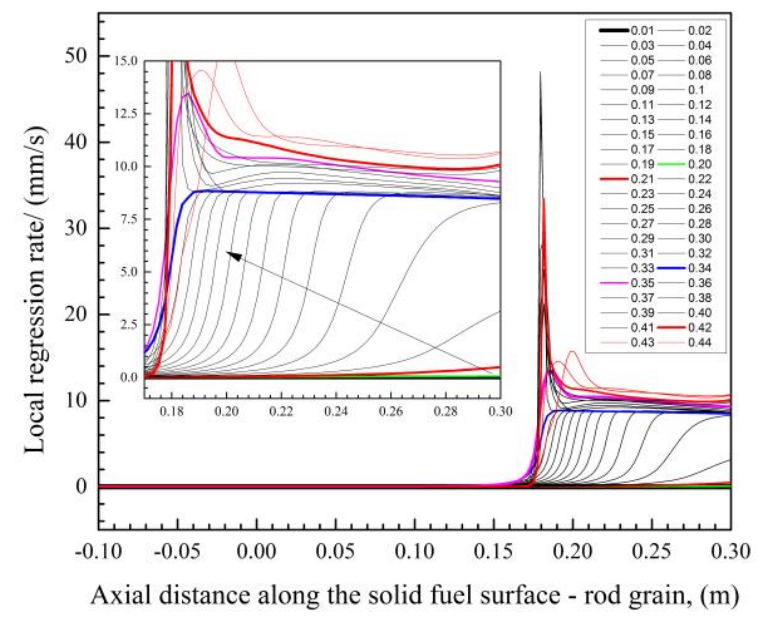

(a)

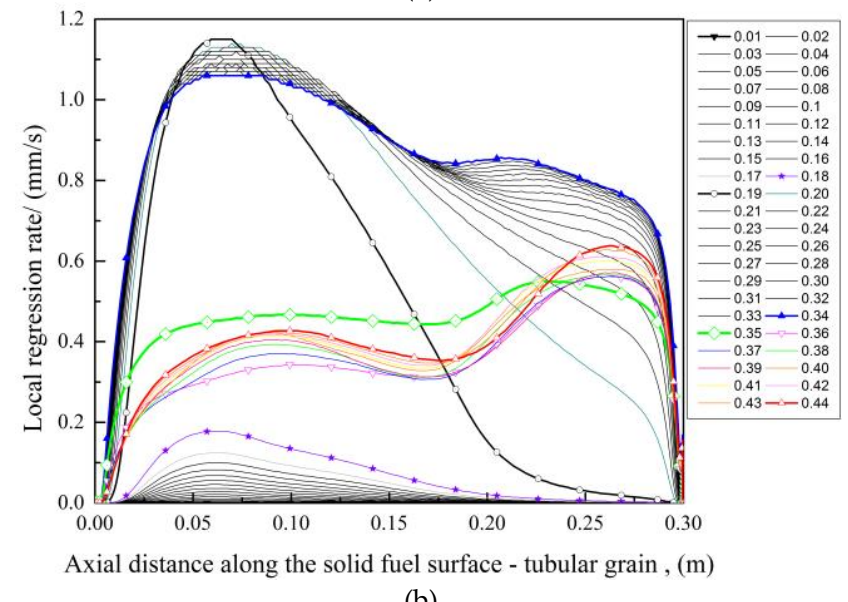

(b)

Fig. 3. Axial distributions of local regression rate for solid fuel surface at different time intervals.

Normally, ignition delay time includes; heating (gasification) time delay, mixing time delay, and chemical processes time delay; but mixing time is very small and can be ignored. Fig. 3 shows the ignition and combustion processes during the whole simulation for the proposed design. Fig. 3a presents the local regression rates for outer surface of the rod solid fuel. As can be seen, the pyrolysis starts from the end of the combustor $(0.3 \mathrm{~m})$ due to the effect of incoming air jet. As time progresses, the pyrolysis moved towards the inlet till the distance $0.17 \mathrm{~m}$. For sustained combustion, the regression rate is significantly much higher than tubular grain surface regression. Fig. $3 \mathrm{~b}$ shows the local regression rates of inner surface of the tubular solid fuel.

Since the proposed design increases the surface are attached with the incoming air the as described above, the heat and mass transport at the solid surface is increased as well as shown in Fig. 4 . In this figure, the heat transferred into the solid fuel surface for the two designs is presented. The proposed design reveals mush higher heat flux than the classic one. This may due to the flow behavior inside the combustion chamber at which the proposed design decreases the flow area and hence increases the mass flux. Further, a new recirculation zone is created at the end of the combustor in the new design.

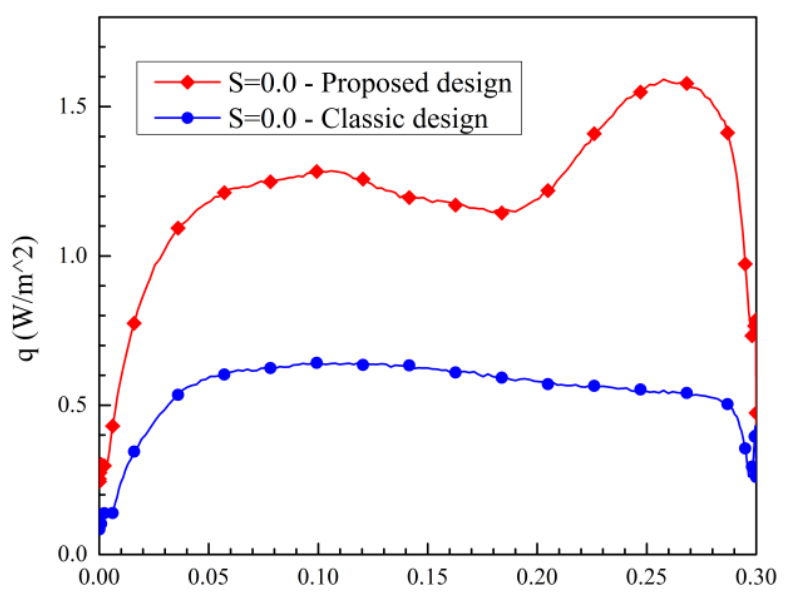

Axial distance along the solid fuel surface - tubular grain , (m) Fig. 4. The local heat flux at the interface along the solid fuel surfaces.

The local regression rate is primarily governed by the heat transport to the solid fuel surface, therefore, same behavior as the local heat transfer is observed for the local regression rate, where for the proposed design, the regression rate increases rapidly in the corner recirculation zone till reaches its first peak at the reattachment point then decreases gradually downstream of the reattachment point and finally increases till reaches its maximum and the second recirculation zone, as shown in Fig. 5.

On comparing the regression rates of the classical and the new designs, the proposed designs have higher regression rate with a new trend. In which, the peak is shifted into the end of the combustor due to the high temperature offered by the combustion of the outer grain (see 
Fig. 5). This can be seen clearly in Fig. 6, where the temperature contour represents the flames (high temperature) produced near to each surface.

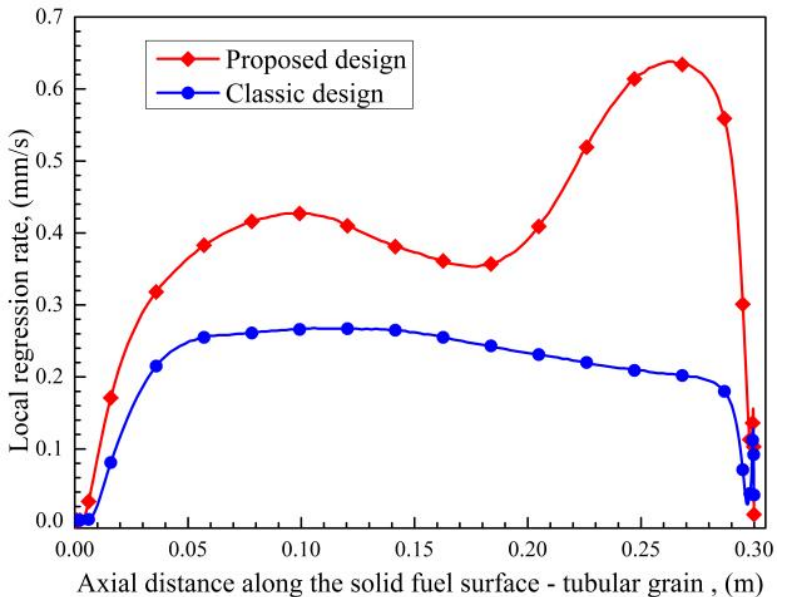

Fig. 5. Regression rates along axial direction for the proposed and the classic design.

The radial temperature profiles at different axial locations along the combustion chamber and afterburning chamber for both designs are presented in Figs. 7 and 8 , respectively.

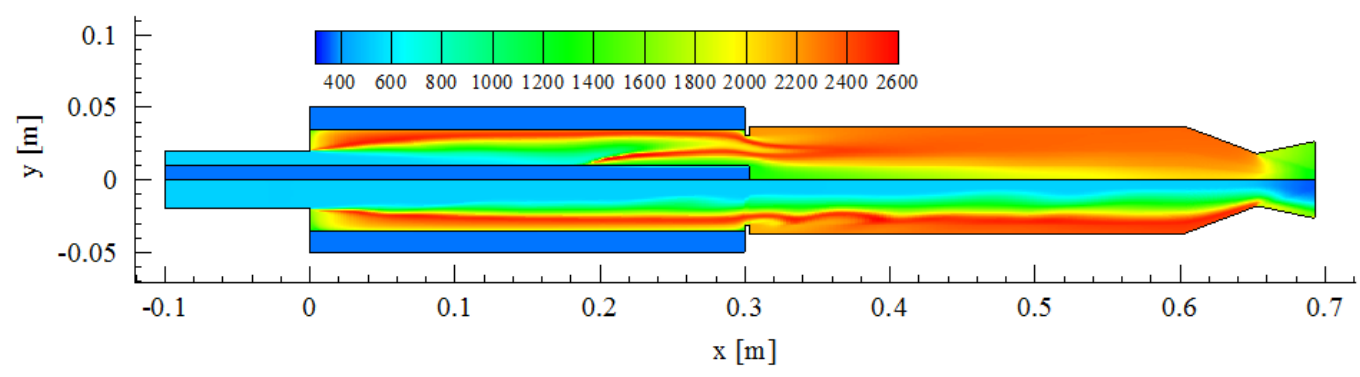

Fig. 6. Temperature contours of the proposed (upper half) and the classic design (lower half) [K].
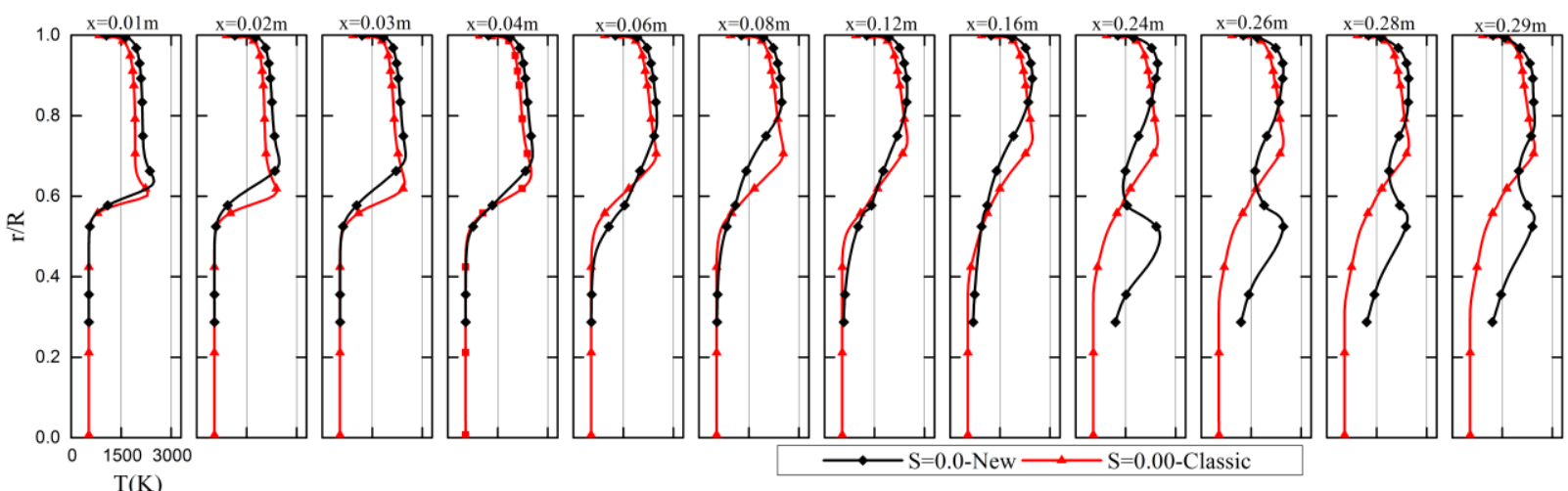

Fig. 7. Radial temperature profiles at different axial positions in SFRJ combustor.

\section{CONCLUSION}

This paper proposed and numerically examined a new design of solid-fuel ramjet motor. An in-house CFD code is developed using FORTRAN language and parallel computing. The developed code is structured, finite-volume, density-based, cell-centered flow solver that
For the classic design, in the first profile, downstream the sudden expansion $(x=0.01 m)$ displays rapid increase of the inlet temperature to its maximum at approximately $r / R=0.61$ (see Fig. 7). Going further downstream till the eighth axial position $(x=0.16 m)$, the peak temperature shifts towards the solid fuel surface $(r / R=1.0)$. The same behavior of the peak temperature is observed for the proposed design with a significant difference that for each axial position the peak temperature goes further close to the wall beside new trend is observed at last four locations at which a new flame is created on the rod solid fuel. Therefore, the proposed design offered two flames inside the combustor then helped to achieve uniform distribution of temperature throughout the engine (see also Figs. 6 and 8). 


\section{proposed design.}

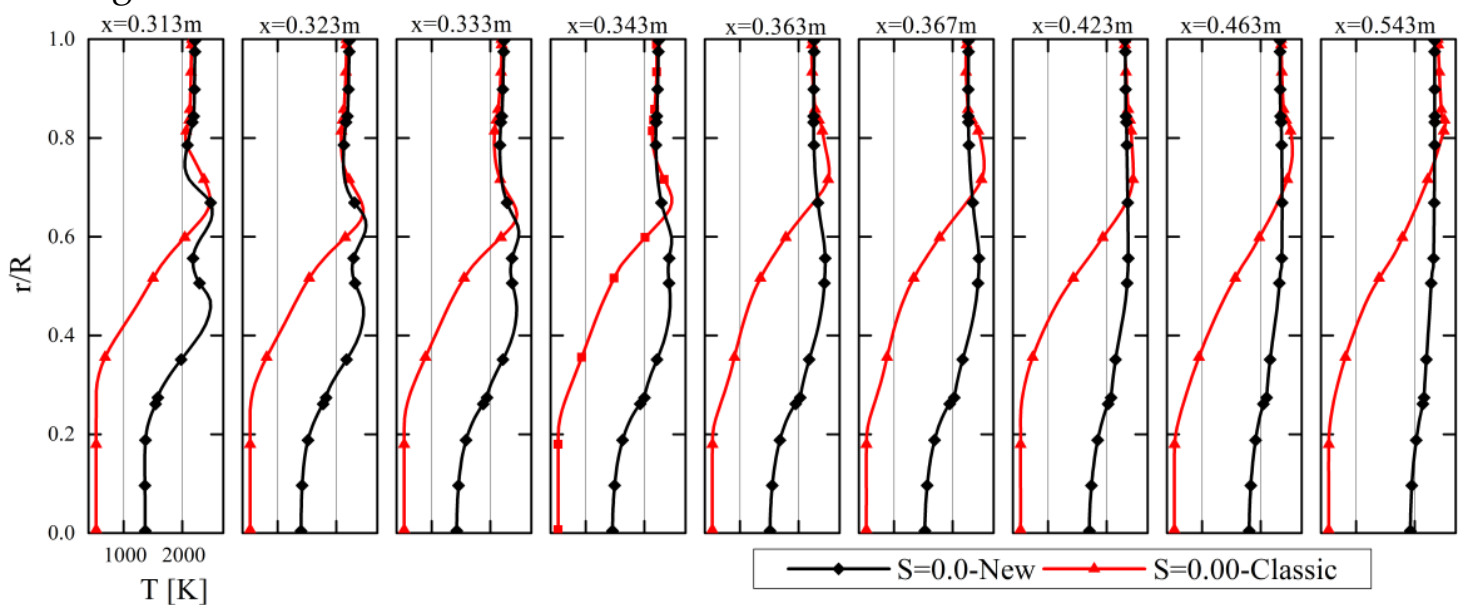

Fig. 8. Radial temperature profiles at different axial positions in SFRJ afterburning chamber.

\section{REFERENCES}

[1] Waltrup, P. J., et al. "History of US Navy ramjet, scramjet, and mixed-cycle propulsion development." Journal of propulsion and power 18.1 (2002): 14-27.

[2] Pelosi-Pinhas, Deborah, and Alon Gany. "Solid-fuel ramjet regulation by means of an air-division valve." Journal of Propulsion and Power 16.6 (2000): 1069-1074.

[3] http://aerostories.free.fr/constructeurs/leduc/page8.html

[4] D. Duesterhaus, A. Hogl, Measurements in a Solid Fuel Ramjet Combustion with Swirl, AIAA Paper (88-3045). doi:10.2514/6.1988-3045.

[5] Lee, C., Na, Y., Lee, J. W., and Byun, Y. H. (2007). “Effect of induced swirl flow on regression rate of hybrid rocket fuel by helical grain configuration. " Aerosp. Sci. Technol., 11(1), 68 -76 .

[6] Li, X., Tian, H., and Cai, G. (2013). "Numerical analysis of fuel regression rate distribution characteristics in hybrid rocket motors with different fuel types. "Sci. China Technol. Sci., 56(7), $1807-1817$.

[7] Gascoin N, Fau G, Gillard P, Mangeot A. Flash Pyrolysis of High Density PolyEthylene, in: 49th AIAA/ASME/SAE/ASEE Joint Propulsion Conference. 2013; 3833.

[8] Mawid M, Sekar B. Kinetic modeling of ethylene oxidation in high speed reacting flows. AIAA Paper 1997;3269.

[9] Baurle R, Mathur T, Gruber M, Jackson K. A numerical and experimental investigation of a scramjet combustor for hypersonic missile applications. AIAA paper 1998;3121:1998.

[10] Stoliarov S I, Walters R N. Determination of the heats of gasification of polymers using differential scanning calorimetry. Polym. Degrad. Stab. 2008;93:422-27.

[11] Kim, Kyu Hong, Chongam Kim, and Oh-Hyun Rho. 2001. Methods for the accurate computations of hypersonic flows: I. AUSMPW+ scheme. Journal of Computational Physics 174.1. 38-80.

[12] Van Leer, Bram. 1979. Towards the ultimate conservative difference scheme. V. A second-order sequel to Godunov's method. Journal of computational Physics 32.1. 101-136.

[13] Van Albada, G. D., Bram Van Leer, and W. W. Roberts Jr. 1997. A comparative study of computational methods in cosmic gas dynamics: Upwind and High-Resolution Schemes. Springer Berlin Heidelberg, 95-103.
[14] Menter, Florian R. 1994. Two-equation eddy-viscosity turbulence models for engineering applications. AIAA journal 32.8. 1598-1605.

[15] Zhang, L. P., and Z. J. Wang. 2004. A block LU-SGS implicit dual time-stepping algorithm for hybrid dynamic meshes. Computers \& fluids 33.7. 891-916.

[16] O. Musa, Z. Changshen, C. Xiong, L. Yingkun. 2016. Verification Study of a CFD-RANS Code for Turbulent Flow at High Reynolds Numbers. International Journal of Modeling and Optimization. 6. 1-10.

[17] O. Musa, Z. Changsheng, C. Xiong, G. Lunkun. 2016. Prediction of swirling cold flow in a solid-fuel ramjet engine with a modified rotation/curvature correction SST turbulence model. Applied Thermal Engineering. 105. 737-754.

[18] O. Musa, C. Xiong, Z. Changsheng, W. Li. 2017. Effect of inlet conditions on swirling turbulent reacting flows in a solid fuel ramjet engine. Applied Thermal Engineering. 113:186-207.

[19] O. Musa, C. Xiong, Z. Changsheng, G. Lunkun. 2016. Assessment of the modified rotation/curvature correction SST turbulence model for simulating swirling reacting unsteady flows in a solid-fuel ramjet engine. Acta Astronautica,. 129. 241-252.

[20] O. Musa, C. Xiong, Z. Chang-sheng, L. Ying-kun, L. Wen-He. Investigations on the influence of swirl intensity on solid-fuel ramjet engine. Computers \& Fluids, Volume 167, 2018, Pages 82-99.

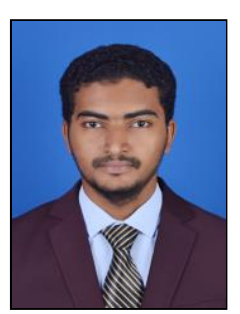

Omer Musa received his bachelor of science degree in mechanical engineering (First Class) from Omdurman Islamic University, Sudan in 2008; the master of science in mechanical engineering (Excellent) from Sudan University of Science and Technology, Sudan in 2013. PhD in mechanical engineering from Nanjing University of Science and Technology, China in 2017. He has been appointed as a lecturer at Omdurman Islamic University, Khartoum, Sudan since 2009. Dr. Musa is holding a position of Assistant Professor at Omdurman Islamic University, Sudan. His research interests include computational fluid dynamics, combustion and propulsion systems. ORCID: 0000-0003-3129-3902 